\title{
Monoamine oxidase A gene promoter methylation and transcriptional downregulation in an offender population with antisocial personality disorder
}

\author{
D. Checknita, G. Maussion, B. Labonté, S. Comai, R. E. Tremblay, F. Vitaro, N. Turecki, A. Bertazzo, \\ G. Gobbi, G. Côté and G. Turecki
}

\begin{abstract}
Background
Antisocial personality disorder (ASPD) is characterised by elevated impulsive aggression and increased risk for criminal behaviour and incarceration. Deficient activity of the monoamine oxidase A (MAOA) gene is suggested to contribute to serotonergic system dysregulation strongly associated with impulsive aggression and antisocial criminality.
\end{abstract}

\section{Aims}

To elucidate the role of epigenetic processes in altered $M A O A$ expression and serotonin regulation in a population of incarcerated offenders with ASPD compared with a healthy non-incarcerated control population.

\section{Method}

Participants were 86 incarcerated participants with ASPD and 73 healthy controls. MAOA promoter methylation was compared between case and control groups. We explored the functional impact of MAOA promoter methylation on gene expression in vitro and blood 5-HT levels in a subset of the case group.

\section{Results}

Results suggest that MAOA promoter hypermethylation is associated with ASPD and may contribute to downregulation of MAOA gene expression, as indicated by functional assays in vitro, and regression analysis with whole-blood serotonin levels in offenders with ASPD.

\section{Conclusions}

These results are consistent with prior literature suggesting MAOA and serotonergic dysregulation in antisocial populations. Our results offer the first evidence suggesting epigenetic mechanisms may contribute to MAOA dysregulation in antisocial offenders.

\section{Declaration of interest}

None.
Antisocial personality disorder (ASPD) is a condition characterised by a persistent pattern of disregard for the rights of others manifesting prior to age 15 as conduct disorder then continuing into adulthood. A core feature of ASPD is an elevated and persistent pattern of impulsive aggression, which places those with the condition at increased risk for criminal offending leading to increased risk for incarceration and recidivism, presenting a significant burden on society and the criminal justice system. As such, ASPD is highly overrepresented in offender populations with upwards of $40 \%$ of inmates meeting diagnostic criteria. ${ }^{1,2}$ ASPD is highly heritable with estimates of heritability reaching as high as $\left(h^{2}=0.80\right) .^{3}$ Considering the pervasive pattern of impulsive aggression emerging early in life among antisocial populations, convergent genetic and environmental risk factors in early childhood are strongly suggested to contribute to impaired ability to suppress aggressive behaviour. ${ }^{4,5}$ Risk factors including severe physical and/or sexual abuse early in life, as well as parental neglect, represent particularly salient predictors of emergent patterns of impulsive aggression contributing to the development of ASPD. ${ }^{4-6}$ These forms of early-life adversity are suggested to impair social learning and enactment of appropriate prosocial responses to perceived threats, resulting in a greater reliance on aggression. ${ }^{7-9}$ A large body of evidence suggests that impulsive aggression is associated with dysfunction of the serotonin (5-hydroxytryptamine, 5-HT) system.

Studies of impulsive aggression in rodents, non-human primates and human forensic populations suggest a system-wide serotonergic dysregulation manifesting in reduced central 5-HT activity. ${ }^{10}$ Altered expression of genes contributing to the regulation of 5-HT have been observed in populations showing high impulsive aggression. ${ }^{10,11}$ Among these genes, the monoamine oxidase A (MAOA) gene (chrX: 43514155-43606071), which encodes the monoamine oxidase (MAO) enzyme that metabolises 5-HT into 5-hydroxyindoleacetic acid (5-HIAA) following reuptake, ${ }^{12}$ has shown a particularly robust association with impulsivity and aggression. ${ }^{10}$ Complete inactivation of MAOA through a rare X-linked point-mutation in the gene's eighth exon among the males in a Dutch family was associated with violent impulsive criminal behaviour, mild intellectual disability and dysregulated 5-HT levels. ${ }^{13}$ Knock-out studies of MAOA in rodents have produced similar aggressive phenotypes. ${ }^{14-16} \mathrm{~A}$ low-expressing variable number tandem repeat (VNTR) in the promoter region of $M A O A$ was associated with increased sensitivity to early-life adversity and risk for antisocial aggression in adulthood. ${ }^{17-20}$ More recently, a longitudinal study spanning 30 years illustrated a moderating role of the low-expressing $M A O A$ variant between exposure to early-life adversity and risk for developing conduct disorder and ASPD. ${ }^{6,21}$ However, attempts to replicate the association between the low-expressing $M A O A$ polymorphism and ASPD risk have yielded inconsistent results. ${ }^{22}$ Other molecular mechanisms that may contribute to downregulation of MAOA in impulsive aggression are not well understood.

Epigenetic investigation has allowed for greater insight into how environmental factors may interact with the genome to facilitate altered gene expression and induce behavioural phenotypes, which in turn increase risk of mental illness. ${ }^{23}$ Studies in animals and humans indicate that early-life adversity is associated with epigenetic changes in expression of genes that are critical to regulate important biological systems, such as the stress response. ${ }^{24,25}$ Recent work suggests that early-life adversity may also contribute to epigenetic alteration of serotonergic genes 
in aggressive populations. For instance, hypermethylation of the serotonin transporter gene (5-HTT) promoter region has been associated with ASPD among women who had experienced sexual abuse in childhood. ${ }^{26,27}$ Similarly, another study illustrated that promoter hypermethylation of the same gene is associated with reduced brain 5 -HT synthesis in childhood aggression. ${ }^{28}$

This suggests that genes critical in regulating 5-HT may be epigenetically regulated in populations prone to high aggression. Recent work in rodents and humans also suggests that $M A O A$ is poised for epigenetic regulation. ${ }^{29}$ In a peripubertal stress model of aggression in rats, enrichment of $\mathrm{H} 3 \mathrm{ac}$ in the MAOA promoter was associated with altered gene expression and highly aggressive behaviour among stressed rats. ${ }^{30}$ The authors also showed treatment with the MAO inhibitor clorgyline led to reduced aggression among rats exposed to stress during the peripubertal period. ${ }^{30}$ Further, MAOA promoter methylation was recently shown to correlate inversely with MAO enzymatic activity in the brains of healthy males. ${ }^{31}$ Together, this work suggests that genes involved in regulating 5-HT may fall under epigenetic influence in antisocial populations, although epigenetic regulation of $M A O A$ has not yet been investigated. As such, the current study explores the potential role of epigenetic regulation of MAOA in a population of incarcerated offenders with ASPD.

\section{Method}

\section{Participants}

The case participants were incarcerated men $(n=86$, mean age 27.1) who met DSM-IV-TR ${ }^{32}$ criteria for ASPD from the Epidemiology of Mental Disorders, Personality Disorders, and Intellectual Disabilities in Prison Settings cohort. In this study, a representative sample of offenders in Quebec who received a federal sentence (i.e. 2 years or more) requiring incarceration were recruited over a 4-year period through the Regional Reception Center at the Correctional Service of Canada complex in Sainte-Anne-des-Plaines. Following recruitment, inmates were assessed for DSM-IV-TR Axis I and II diagnoses using the Structured Clinical Interview (SCID). ${ }^{33}$ Sociodemographic and judicial information was gathered. Following psychiatric and sociodemographic assessments, participants were asked to provide a blood sample. Following informed consent, whole blood samples were obtained and provided the basis for downstream epigenetic and analytic chemistry experiments. As a result of the high rate of comorbidity representative of offender populations with ASPD, ${ }^{1,2}$ participants with Axis I and II comorbidities were also included into the study (online Table DS1).

Control participants were healthy non-incarcerated individuals $(n=73)$ derived from the Québec Longitudinal Study of Kindergarten Children (QLSKC) $)^{34}$ and gender- and age-matched to the case participants. Briefly, the QLSKC cohort consists of 3018 participants initially recruited from kindergarten classes in French-speaking schools across the province at the age of 6 . Random and proportional recruitment according to the 11 administrative districts of the province yielded a representative sample of the Quebec population. Multiple behavioural and psychiatric assessments are available from this cohort at different time points during development as well as adulthood. ${ }^{35}$ For the current study, we selected a random sample of QLSKC participants who had no DSM-III-R Axis I and II diagnoses ${ }^{36}$ at any assessment point, and whose aggression scores did not deviate from the population mean.

The study was approved by the research ethics boards of the University of Montreal, McGill University, Université du Québec à Trois-Rivières, Institut Philippe-Pinel de Montréal, and
Correctional Services Canada at both provincial and federal levels. Written informed consent was obtained from all participants.

\section{Analysis of MAOA promoter methylation}

DNA was extracted from whole blood using QIAGEN's QIamp DNA Mini Kit according to the manufacturer's instructions (Qiagen, Germantown, Maryland, USA; www.qiagen.com). The extracted genomic DNA was then bisulfite-treated using QIAGEN's Epitect Bisulfite Kit following manufacturer guidelines. Methylation analysis of a region within the MAOA promoter spanning $\sim 1.5 \mathrm{~kb}$ (chrX: 43514507-43515991) comprised of 71 CpGs was performed using Sequenom's EpiTYPER at Genome Quebec's Innovation Centre.

\section{In vitro functional analysis of MAOA promoter methylation and gene expression}

A $678 \mathrm{bp}$ region of the MAOA promoter was cloned into the pCpG free-basic Lucia vector using primer sequences: Forward 5'-TATA GGATCC CGGGTATCAGCTGAAACATCA-3' and Reverse 5' - TATA AAGCTT GGTGATTCGACCTCAAGAGAGT-3' containing BAMH1 and HINDIII restriction sites, respectively (underlined in sequences). The addition of BAMH1 and HINDIII restriction sites in the primers ensured that the region of interest (ROI) was cloned into the vector in correct orientation relative to the Lucia reporter gene. The plasmid was then submitted to Sss1 methylase (New England Biolabs, Ipswich, Massachusetts, USA; www.neb.ca) treatment involving a $4 \mathrm{~h}$ incubation period at $37^{\circ} \mathrm{C}$. This process facilitates the addition of a methyl group to each of the $16 \mathrm{CpG}$ sites within the ROI, thus generating a fully methylated construct. HEK293 cells were seeded in 24 well-plates for a period of $24 \mathrm{~h}$. Following this period, native vector, unmethylated and methylated constructs were co-transfected with pGL3 control vector used for normalisation in HEK293 cells for an additional $24 \mathrm{~h}$. The impact of native vector, unmethylated and methylated constructs on luciferase reporter gene activity in cell-extract and cell media was quantified by Berthold Luminometer using a dual-luciferase assay reporter kit (Promega, Madison, Wisconsin, USA; www.promega.com). Data were collected using Simplicity 4.2 software for Windows XP.

\section{Analysis of 5-HT serum levels in blood}

Analysis of 5-HT serum levels in blood was available for a subsample of 80 case participants. It was performed using a high-pressure liquid chromatography (HPLC) system (Shimadzu LC-10AD, Columbia, Maryland, USA; www.shimadzu.com) coupled to a fluorometric detector (Shimadzu RF-10AXL) according to the method of Comai et al. ${ }^{37}$ Briefly, HPLC analysis of 5-HT relied on selective fluorometric detection via an online HPLC retention of the protein fraction in a precolumn system and subsequent elution via isocratic gradient phosphate buffer (0.004 M, pH 3.5)/acetonitrile (80/20, v/v). The separation was performed at a flow rate of $1 \mathrm{~mL} / \mathrm{min}$ using an analytical Platinum EPS C18 100A column $(5 \mu \mathrm{m} ; 250 \times 4.6 \mathrm{~mm}$; Alltech, Deerfield, Illinois, USA; www.alltech.com). The fluorometric detector was set at the excitation and emission wavelengths of 285 and $345 \mathrm{~nm}$, respectively.

\section{Statistical analyses}

Statistical analyses were performed using SPSS software version 20 for Windows 8. Analysis of MAOA promoter methylation differences between case and control participants was performed by two-way mixed-model ANOVA with post hoc least significant 
difference (LSD) correction for multiple comparisons. Results from this analysis also provided the basis for subsequent functional analysis. To determine the relative in vitro impact of native vector, unmethylated and methylated constructs on luciferase reporter gene activity, data were analysed using a oneway ANOVA with Bonferroni post hoc correction for multiple comparisons. Finally, multiple regression analysis was used to determine whether $M A O A$ promoter $\mathrm{CpG}$ methylation, as measured by EpiTYPER, was associated with variance in serum 5-HT levels in a subset of 80 case participants.

\section{Results}

\section{MAOA promoter methylation}

To assess the MAOA methylation status in our groups, we investigated a $1.5 \mathrm{~kb}$ region of the promoter region. This region contains a total of $71 \mathrm{CpGs}$, and was selected to gain maximum coverage of the MAOA promoter region. It also included a $466 \mathrm{bp}$ region where methylation has previously been correlated with MAO enzymatic function in the brain. ${ }^{31}$ We first extracted DNA from whole blood then bisulfite-treated the DNA in preparation for methylation analysis. For methylation mapping, we used EpiTYPER, which is a method that uses uracil-specific enzymatic base cleavage of bisulfite treated DNA followed by a mass spectrometry based quantification of methylation with single CpG dinucleotide resolution. A two-way mixed-model ANOVA revealed a significant main effect of group $(F(1,1650)=16.866$, $P=0.000042)$, as well as a significant main effect of $\mathrm{CpG}$ site $(F(52,365)=567.5, \quad P<0.001)$ and a significant interaction between group and $\mathrm{CpG}$ site $(\mathrm{F}(52,365)=6.617, P<0.001)$. More specifically, our results indicated significant overall hypermethylation of the MAOA promoter region among the case group compared with the control group (Fig. 1(a)). Post hoc LSD analysis for multiple-testing revealed significant group differences in methylation levels for 34 of the 71 MAOA promoter CpGs assessed by EpiTYPER, 31 of which were hypermethylated among the case participants (Fig. 1(b) and (c)).

\section{Functional assessment}

To analyse the potential functional impact of the MAOA promoter hypermethylation observed in the case group on gene transcription, we cloned a $678 \mathrm{bp}$ ROI into a CpG-free promoterless vector (pCpGfree-Basic Vector). Selection of this $678 \mathrm{bp}$ (chrX: 43515313-43515991) ROI within the MAOA promoter for functional assays was based on the following. In silico analysis using Transfac revealed several predicted binding domains for transcription factors based on transcription factor consensus sequence (online Table DS2). As such, methylation in this region is more likely to affect binding of transcription factors leading to altered transcriptional activity of MAOA. Second, this ROI represented the region showing the most pronounced differences in $\mathrm{CpG}$ methylation between the case and control groups (Fig. 2). Thus, the ROI represented a strong candidate for in vitro functional assays. Finally, methylation within a $466 \mathrm{bp}$ region of the selected ROI has been previously correlated to brain MAO enzymatic levels in healthy human participants by others. ${ }^{31}$

Native vector, unmethylated, and fully-methylated ROI constructs were produced and transfected into HEK293 cells for a period of $24 \mathrm{~h}$. Following this period, the impact of each construct on luciferase reporter gene activity was quantified. A one-way ANOVA analysis was used to compare the effect of native vector, unmethylated and fully methylated constructs on reporter gene activity. Analysis revealed a significant between-group effect $(F(2,11)=1206.9, \quad P<0.0001)$. The unmethylated construct showed a 12 -fold increase of luciferase reporter activity (23.90, s.d. $=0.60)$ compared with the native vector construct $(1.95$, s.d. $=0.07)\left(P=9.2 \times 10^{-14}\right)$. The methylated construct showed a significant $53 \%$ decrease in reporter activity $(11.19$, s.d. $=0.39)$ compared with the unmethylated construct $\left(P=5.9 \times 10^{-10}\right)$ (Fig. 3). Thus, our results suggest that the selected 678 bp ROI has clear promoter activity, and that methylation in this region leads to a significant decrease in this activity.

As the functional study results suggested that methylation of our ROI may be contributing to downregulation of MAOA gene expression in our case group, we sought to determine whether $M A O A$ promoter methylation would be significantly associated with blood 5-HT levels in these participants. To this end, we assessed 5-HT blood serum levels using HPLC. Regression analysis indicated that methylation at $45 \mathrm{CpGs}$ in the $M A O A$ promoter was associated with blood 5-HT levels, and explained $88.8 \%$ of its variance $\left(R^{2}=0.888, F(44,13)=2.34, P=0.048\right)$. Our analysis suggested that increased MAOA promoter methylation was positively associated with 5-HT levels in blood. These results suggest that MAOA promoter methylation may play a role in 5-HT dysregulation among offenders with ASPD.

\section{Discussion}

\section{Main findings}

Deficient activity of the MAOA gene has been frequently associated with increased impulsivity and aggression. Work spanning the past decade has suggested that a low expressing functional polymorphism of $M A O A$ may play a mediating role between early-life adversity and the development of ASPD., ${ }^{6,17,20-22}$ However, efforts to replicate these findings have yielded inconsistent and inconclusive results. ${ }^{22}$ As such, this study sought to explore the potential impact of epigenetically altered expression of MAOA, offering novel insight into molecular mechanisms contributing to dysregulated MAOA expression in a population of offenders with ASPD. Our results suggest that hypermethylation in the $M A O A$ promoter, particularly in its sequence proximal to the transcription start site, is associated with ASPD and may contribute to a downregulation of MAO activity and increased 5-HT levels, a finding consistent with prior work among antisocial offenders. $^{21}$

Dysregulation of 5-HT is strongly associated with elevated impulsive aggression. ${ }^{10}$ This association represents one of the most consistently reported associations between biological factors and behavioural phenotypes acting as risk factors for mental illness. ${ }^{10}$ Typically, reduced 5-HT activity in the central nervous system is linked to elevated impulsive aggression. ${ }^{10}$ Our results suggest that increased MAOA promoter methylation results in decreased MAO production, and increased 5-HT levels in blood serum among offenders with ASPD. This finding is consistent with previous literature indicating decreased MAOA activity among antisocial offenders and elevated peripheral 5-HT in aggression. ${ }^{38}$ Several studies suggest reduced central 5-HT correlates with increased peripheral 5-HT levels in blood platelets in behavioural phenotypes including increased aggression. ${ }^{38-41}$ Although the biological mechanisms underlying this inverse relationship are not yet understood, this phenotype may be suggestive of a broader system-wide dysregulation of 5-HT observable in central and peripheral pathways underlying aggression and impulsivity. ${ }^{38}$

Alterations in DNA methylation are associated with early-life adversity and are thought to be aetiologically related to development of psychopathology, including mood disorders and suicide, commonly observed among individuals who have been exposed to difficult early-life environments. ${ }^{42-44}$ The possibility 


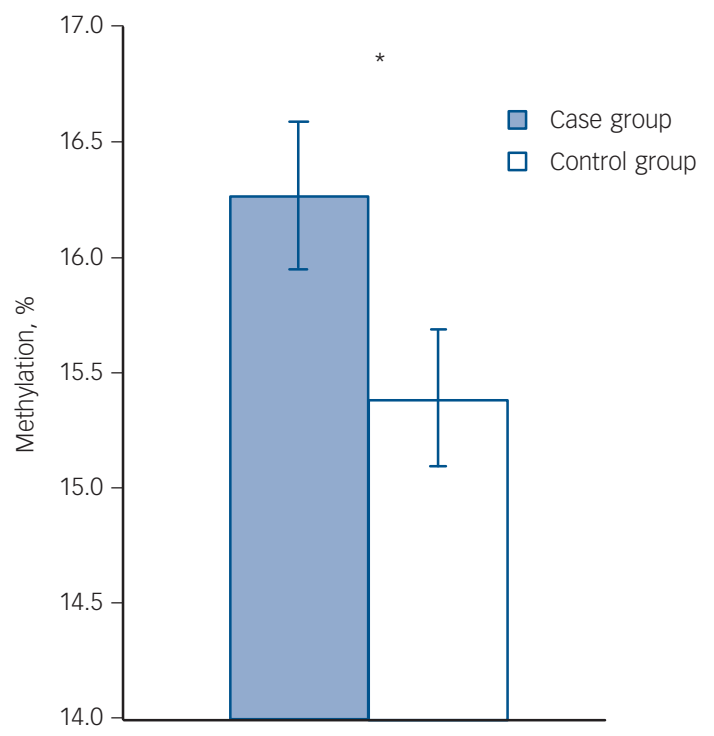

(b)

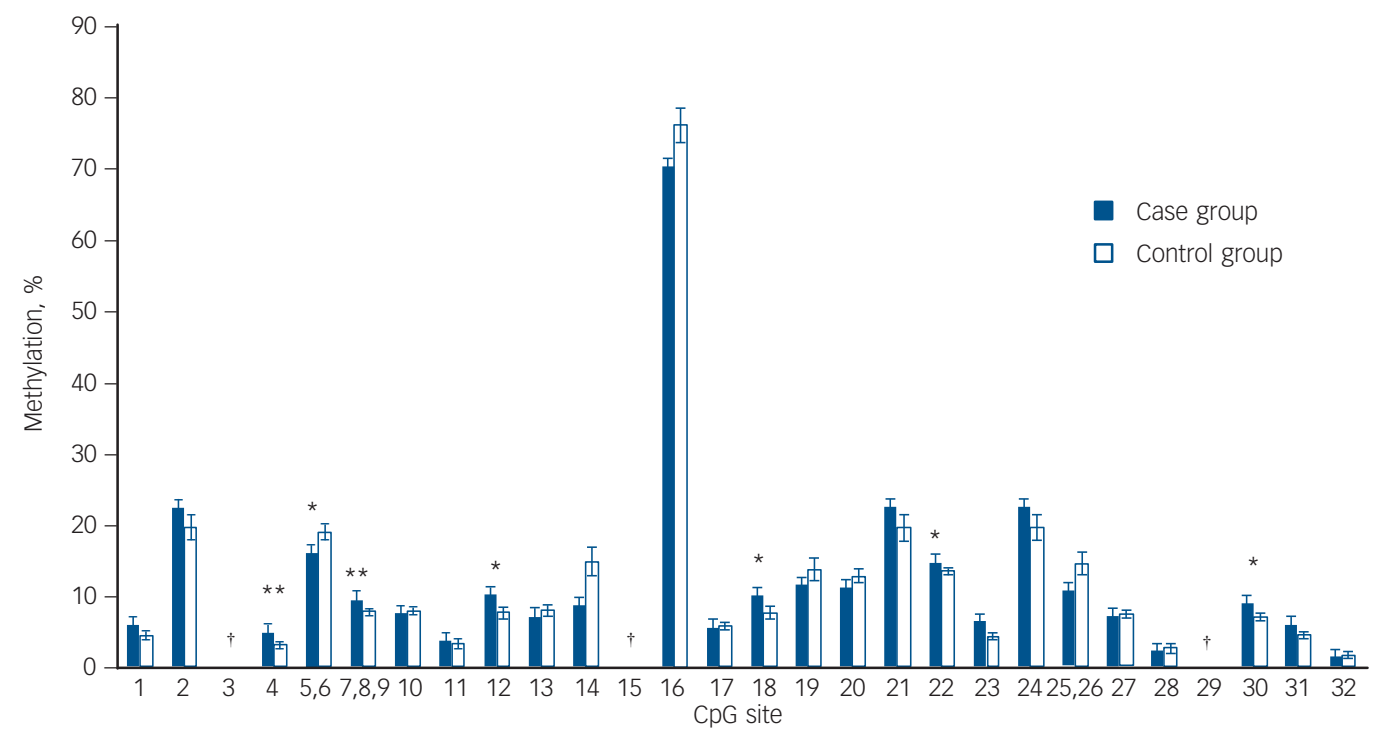

(c)

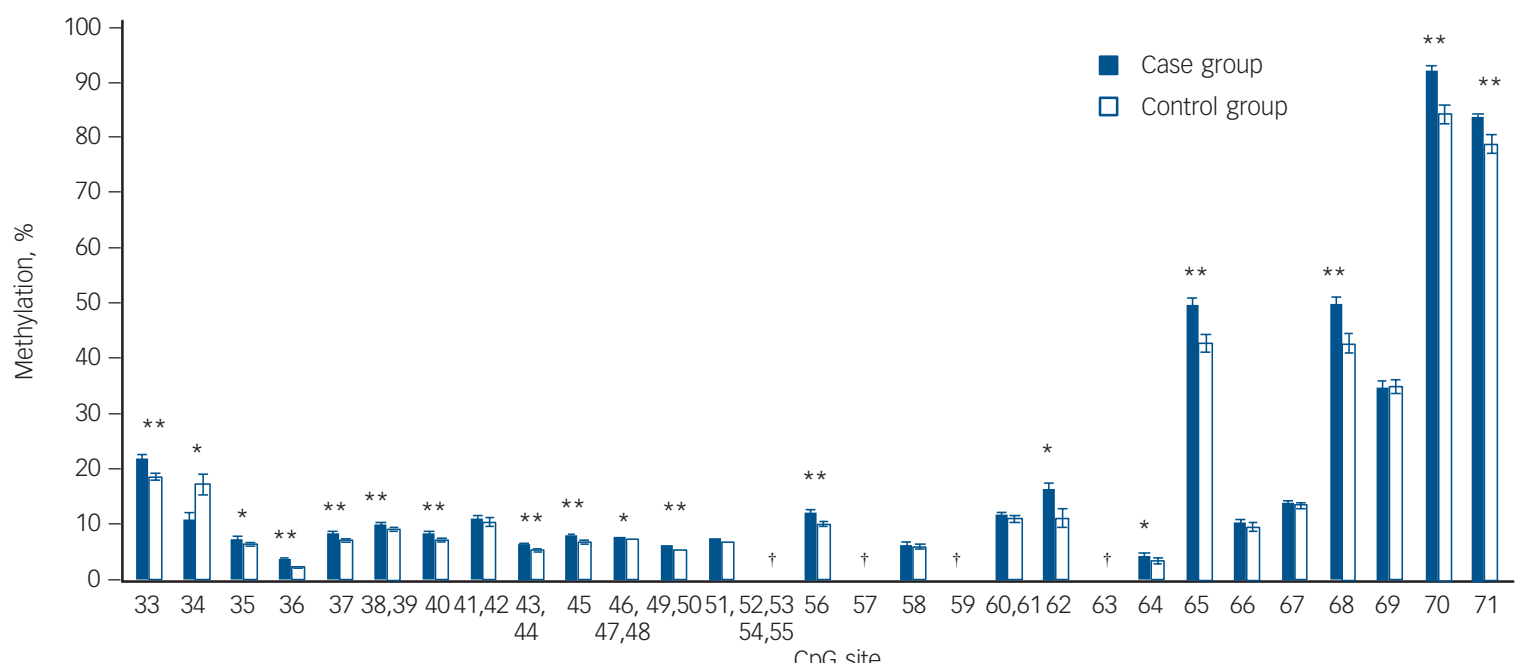

Fig. 1 Methylation of monoamine oxidase A (MAOA) promoter region assessed by EpiTYPER.

(a) Mean methylation for $1.48 \mathrm{~kb}$ region of MAOA promoter. Results indicate significant hypermethylation among the case group compared with the control group $(P<0.001)$.

(b) Methylation profile for CpGs 1-32 of the MAOA promoter region. Results indicate significant group differences in methylation at 7 sites representing 11 CpGs ( $* P<0.05$, $* * P<0.005$. (c) Methylation profile for CPGs 33-71 of the MAOA promoter region. Results indicate significant group differences in methylation at 18 sites representing $23 \mathrm{CpGs}\left({ }^{\star} P<0.05\right.$, $* \star P<0.005$. † Data unavailable. 


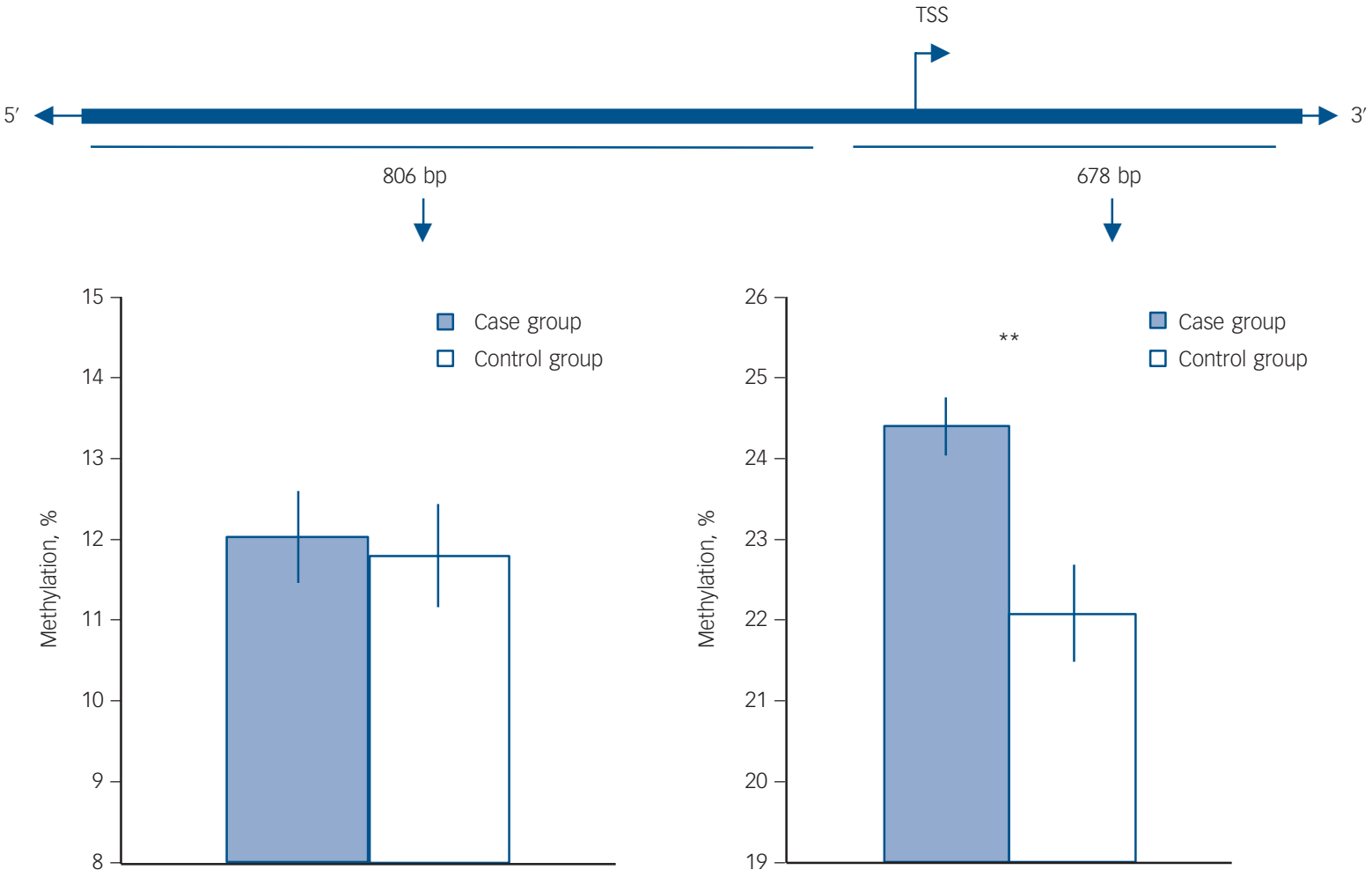

Fig. 2 Comparison of group mean methylation differences between the region of interest (ROI) and pre-ROI regions of monoamine oxidase A (MAOA) promoter relative to the transcription start site (TSS).

Results suggest no significant group difference in pre-ROI CpG methylation (bottom left) and significant hypermethylation in the post-ROI region among the case group compared with the control group $(P<0.005)$ (bottom right).

that MAOA promoter hypermethylation is associated with earlylife adversity is consistent with prior literature illustrating that associations between $M A O A$ sequence variants and childhood maltreatment confer risk for antisocial behaviour. Epigenetic alteration of genes involved in serotonergic regulation is also associated with aggression. Recent work has indicated that hypermethylation of the serotonergic transporter gene (5-HTT) promoter is associated with the development of ASPD among individuals who had experienced sexual abuse during childhood. ${ }^{28,45,46}$ Results from the same group also suggest that the

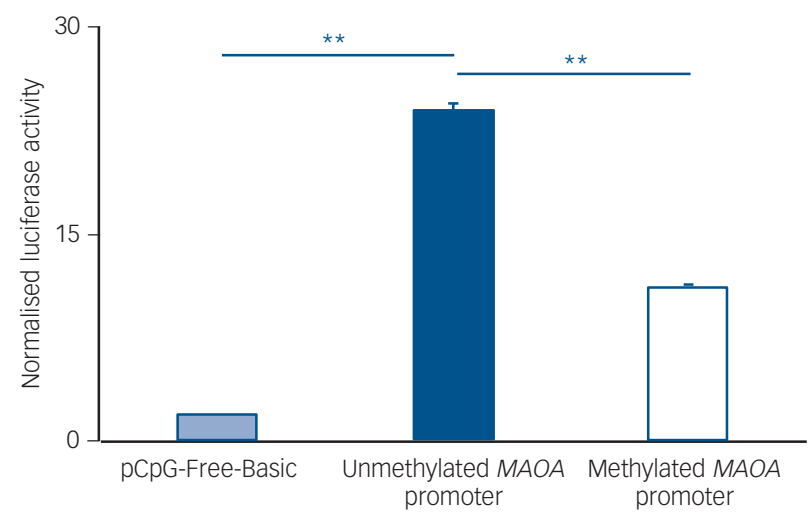

Fig. 3 Luciferase assay comparing transcriptional activity driven by methylated, unmethylated and empty $\mathrm{PCpG}$-free-basic vector monoamine oxidase $A$ (MAOA) region of interest (ROI) constructs.

Results indicate significantly lower transcriptional activity for the methylated construct and empty PGPG-free-basic constructs compared with the unmethylated MAOA RO construct $\left({ }^{*} P<0.005\right)$ magnitude of 5-HTT promoter methylation change relative to controls positively correlates with the presence of parental psychopathology. ${ }^{26}$ Recent work has also indicated that markers of chromatin remodelling may also contribute to altered $M A O A$ expression in aggression. ${ }^{30}$ Together, these studies offer further support of a role for epigenetically altered regulation of 5-HT genes and highlight the importance of pre- and perinatal environmental factors as potential catalysts. Future work should further examine the potential role early-life adversity plays in epigenetic modulation of MAOA.

\section{Limitations}

Limitations of this study include the unavailability of peripheral 5-HT measures among the control group and measures of MAO enzymatic levels for both group. As such, inference of a direct causal relationship between MAOA promoter hypermethylation and altered MAO and 5-HT activity between case and control groups cannot be made. However, our results are consistent with such a possibility, as well as with prior work suggesting a direct relationship between peripheral MAOA promoter methylation and positron emission tomography estimated MAO enzymatic activity in the brain of healthy men. ${ }^{31}$ Further, our experimental design prohibits us from determining whether the MAOA promoter methylation observed is directly attributable to ASPD or to environmental antecedents such as early-life adversity. Inclusion of a group with ASPD without presence of early-life adversity could help to clarify this relationship in subsequent studies. Since brain tissue cannot be obtained from living participants, whole blood was used as a proxy tissue for methylation analysis. Although the relationship between central and peripheral methylation patterns is not yet understood, recent 
studies have illustrated associations between methylation patterns in peripheral tissue and antisocial phenotypes. ${ }^{28,45,46}$ Finally, ASPD is a developmental condition typified by significant psychiatric comorbidity. As such, the specificity of our results to ASPD may be limited by the retrospective case-control design of the study, although there is a considerable amount of evidence supporting this link in the literature. Future studies should explore the specificity of MAOA promoter methylation and ASPD.

\section{Implications}

To our knowledge, the current study presents the first evidence suggesting epigenetic mechanisms may play a functional role in modulating MAOA expression and regulating 5-HT levels in a population of offenders with ASPD. Thus, the results presented offer crucial insight into molecular mechanisms underlying impulsive aggression, a phenotype also linked to increased risk for mental illness. It is our hope that the current study provides a foundation for understanding the role of epigenetic processes in the biology of aggression.

D. Checknita, MSc, G. Maussion, PhD, McGill Group for Suicide Studies, Douglas Mental Health University Institute, McGill University, Montreal, Canada; B. Labonté PhD, Fishberg Department of Neuroscience and Friedman Brain Institute, Icahn School of Medicine at Mount Sinai, New York, USA; S. Comai, PhD, Neurobiological Psychiatry Unit, Department of Psychiatry, McGill University, Montreal, Canada; R. E. Tremblay, PhD, School of Public Health, Physiotherapy and Population Science, University College, Dublin, Ireland, and Departments of Pediatrics and Psychology, University of Montreal, Montreal, Canada; F. Vitaro, PhD, School of Psycho-Education, University of Montreal, Montreal, Canada; N. Turecki, McGill Group for Suicide Studies, Douglas Mental Health University Institute, McGill University, Montreal, Canada; A. Bertazzo, PhD, Department of Pharmaceutical Sciences, Univerity of Padua, Padua, Italy; G. Gobbi, MD, PhD, Neurobiological Psychiatry Unit, Department of Psychiatry, McGill University, Montreal, Canada; G. Côté, PhD, Institute Philippe-Pinel, Department of Psychology, Université de Québec à Trois-Rivères, Montreal, Canada; G. Turecki, MD, PhD, McGill Group for Suicide Studies, Douglas Mental Health University Institute, McGill University, Montreal, Canada

Correspondence: Gustavo Turecki, McGill Group for Suicide Studies, Douglas Mental Health University Institute, Bureau F-3125, 6875 boulevard LaSalle, H4H 1R3, Montreal, Canada. Email: gustavo.turecki@mcgill.ca

First received 15 Jan 2014, final revision 23 Jul 2014, accepted 5 Aug 2014

\section{References}

1 Black DW, Gunter T, Loveless P, Allen J, Sieleni B. Antisocial personality disorder in incarcerated offenders: psychiatric comorbidity and quality of life. Ann Clin Psychiatry 2010; 22: 113-20.

2 Gunter TD, Arndt S, Wenman G, Allen J, Loveless P, Sieleni B, et al. Frequency of mental and addictive disorders among 320 men and women entering the Iowa prison system: use of the MINI-Plus. J Am Acad Psychiatry Law 2008; 36: 27-34.

3 Bornovalova MA, Hicks BM, lacono WG, McGue, M. Familial transmission and heritability of childhood disruptive disorders. Am J Psychiatry 2010; 167 1066-74.

4 Tremblay RE. Developmental origins of disruptive behaviour problems: the "original $\sin ^{\prime}$ hypothesis, epigenetics and their consequences for prevention. J Child Psychol Psychiatry 2010; 51: 341-67.

5 Goldstein RB, Grant BF, Ruan WJ, Smith SM, Saha TD. Antisocial personality disorder with childhood- vs. adolescence-onset conduct disorder: results from the National Epidemiologic Survey on Alcohol and Related Conditions. J Nerv Ment Dis 2006; 194: 667-75.

6 Fergusson DM, Boden JM, Horwood LJ, Miller AL, Kennedy MA. MAOA, abuse exposure and antisocial behaviour: 30-year longitudinal study. Br J Psychiatry 2011; 198: 457-63.

7 Côté SM, Vaillancourt T, LeBlanc JC, Nagin DS, Tremblay RE. The development of physical aggression from toddlerhood to pre-adolescence: a nation wide longitudinal study of Canadian children. J Abnorm Child Psychol 2006; 34: 71-85

8 Petitclerc A, Boivin M, Dionne G, Zoccolillo M, Tremblay RE. Disregard for rules: the early development and predictors of a specific dimension of disruptive behavior disorders. J Child Psychol Psychiatry 2009; 50: 1477-84.
9 Boivin M, Brendgen M, Vitaro F, Forget-Dubois N, Feng B, Tremblay RE, et al. Evidence of gene-environment correlation for peer difficulties: disruptive behaviors predict early peer relation difficulties in school through genetic effects. Dev Psychopathol 2013; 25: 79-92.

10 Comai S, Tau M, Gobbi G. The psychopharmacology of aggressive behavior: a translational approach: part 1: neurobiology. J Clin Psychopharmacol 2012; 32: 83-94.

11 Pavlov KA, Chistiakov DA, Chekhonin VP. Genetic determinants of aggression and impulsivity in humans. J Appl Genet 2012; 53: 61-82.

12 Shih JC, Grimsby J, Chen K, Zhu QS. Structure and promoter organization of the human monoamine oxidase A and B genes. J Psychiatry Neurosci 1993: 18: $25-32$.

13 Brunner HG, Nelen M, Breakefield XO, Ropers HH, van Oost BA. Abnormal behavior associated with a point mutation in the structural gene for monoamine oxidase A. Science 1993; 262: 578-80.

14 Cases O, Seif I, Grimsby J, Gaspar P, Chen K, Pournin S, et al. Aggressive behavior and altered amounts of brain serotonin and norepinephrine in mice lacking MAOA. Science 1995; 268: 1763-6.

15 Vitalis T, Cases O, Callebert J, Launay JM, Price DJ, Seif I, et al. Effects of monoamine oxidase A inhibition on barrel formation in the mouse somatosensory cortex: determination of a sensitive developmental period. J Comp Neurol 1998; 393: 169-84.

16 Scremin OU, Holschneider DP, Chen K, Li MG, Shih JC. Cerebral cortical blood flow maps are reorganized in MAOB-deficient mice. Brain Res 1999; 824: $36-44$.

17 Caspi A, McClay J, Moffitt TE, Mill J, Martin J, Craig IW, et al. Role of genotype in the cycle of violence in maltreated children. Science 2002; 297: 851-4.

18 Haberstick BC, Lessem JM, Hopfer CJ, Smolen A, Ehringer MA, Timberlake D, et al. Monoamine oxidase $A(M A O A)$ and antisocial behaviors in the presence of childhood and adolescent maltreatment. Am J Med Genet B Neuropsychiatr Genet 2005; 135B: 59-64.

19 Kim-Cohen J, Caspi A, Taylor A, Williams B, Newcombe R, Craig IW, et al. MAOA, maltreatment, and gene-environment interaction predicting children's mental health: new evidence and a meta-analysis. Mol Psychiatry 2006; 11: 903-13.

20 Huizinga D, Haberstick BC, Smolen A, Menard S, Young SE, Corley RP, et al. Childhood maltreatment, subsequent antisocial behavior, and the role of monoamine oxidase A genotype. Biol Psychiatry 2006; 60: 677-83.

21 Fergusson DM, Boden JM, Horwood $\amalg$, Miller A, Kennedy MA. Moderating role of the MAOA genotype in antisocial behaviour. Br J Psychiatry 2012; 200: 116-23.

22 Haberstick BC, Lessem JM, Hewitt JK, Smolen A, Hopfer CJ, Halpern CT, et al. $M A O A$ genotype, childhood maltreatment, and their interaction in the etiology of adult antisocial behaviors. Biol Psychiatry 2014; 75: 25-30.

23 Szyf, M. The early life social environment and DNA methylation: DNA methylation mediating the long-term impact of social environments early in life. Epigenetics 2011; 6: 971-8.

24 Zhang TY, Labonté B, Wen XL, Turecki G, Meaney MJ. Epigenetic mechanisms for the early environmental regulation of hippocampal glucocorticoid receptor gene expression in rodents and humans. Neuropsychopharmacology 2013; 38: 111-23.

25 Turecki G, Ernst C, Jollant F, Labonté B, Mechawar N. The neurodevelopmental origins of suicidal behavior. Trends Neurosci 2012; 35: 14-23.

26 Beach SRH, Brody GH, Gunter TD, Packer $\mathrm{H}$, wernett $\mathrm{P}$, Philibert RA. Child maltreatment moderates the association of MAOA with symptoms of depression and antisocial personality disorder. J Fam Psychol 2010; 24: $12-20$.

27 Beach SRH, Brody GH, Todorov AA, Gunter TD, Philibert RA. Methylation at SLC6A4 is linked to family history of child abuse: an examination of the lowa Adoptee sample. Am J Med Genet B Neuropsychiatr Genet 2010; 153B: 710-3.

28 Wang D, Szyf M, Benkelfat C, Provencal N, Turecki G, Caramaschi D, et al. Peripheral SLC6A4 DNA methylation is associated with in vivo measures of human brain serotonin synthesis and childhood physical aggression. PLOS One 2012; 7: e39501.

29 Shumay E, Fowler JS. Identification and characterization of putative methylation targets in the MAOA locus using bioinformatic approaches. Epigenetics 2010; 5: 325-42.

30 Márquez C, Poirier GL, Cordero MI, Larsen MH, Groner A, Marquis J, et al. Peripuberty stress leads to abnormal aggression, altered amygdala and orbitofrontal reactivity and increased prefrontal MAOA gene expression. Transl Psychiatry 2013; 3: e126.

31 Shumay E, Logan J, Volkow ND, Fowler JS. Evidence that the methylation state of the monoamine oxidase A (MAOA) gene predicts brain activity of MAO A enzyme in healthy men. Epigenetics 2012; 7: 1151-60. 
32 American Psychiatric Association. Diagnostic and Statistical Manual of Mental Disorders (4th edn, text revision) (DSM-IV-TR). APA, 2000.

33 First MB, Spitzer RL, Gibbon M, Williams JBW. Structured Clinical Interview for DSM-IV-TR Axis I Disorders: Research Version, Patient Edition (SCID-I/P). Biometrics Research Department, New York State Psychiatric Institute, 2002.

34 Rouquette A, Côté SM, Pryor LE, Carbonneau R, vitaro F, Tremblay RE, et al. Cohort profile: the Quebec Longitudinal Study of Kindergarten Children (QLSKC). Int J Epidemiol 2014; 43: 12-33.

35 Ernst C, Wanner B, Brezo J, Vitaro F, Tremblay R, Turecki G. A deletion in tropomyosin-related kinase $\mathrm{B}$ and the development of human anxiety. Biol Psychiatry 2011; 69: 604-7.

36 American Psychiatric Association. Diagnostic and Statistical Manual of Mental Disorders (3rd edn, revised) (DSM-III-R). APA, 1987.

37 Comai S, Cavalletto L, Chemello L, Bernardinello E, Ragazzi E, Costa CV, et al. Effects of PEG-interferon alpha plus ribavirin on tryptophan metabolism in patients with chronic hepatitis C. Pharmacol Res 2011; 63: 85-92.

38 Moffitt TE, Brammer GL, Caspi A, Fawcett JP, Raleigh M, Yuwiler A, et al. Whole blood serotonin relates to violence in an epidemiological study. Biol Psychiatry 1998; 43: 446-57.

39 Ursinus WW, Bolhuis JE, Zonderland JJ, Rodenburg TB, de Souza AS, Koopmanschap RE, et al. Relations between peripheral and brain serotonin measures and behavioural responses in a novelty test in pigs. Physiol Behav 2013; 118C: 88-96.
40 Askenazy F, Caci H, Myquel M, Darcourt G, Lecrubier Y. Relationship between impulsivity and platelet serotonin content in adolescents. Psychiatry Res 2000; 94: 19-28.

41 Bianchi M, Moser C, Lazzarini C, Vecchiato E, Crespi F. Forced swimming test and fluoxetine treatment: in vivo evidence that peripheral $5-\mathrm{HT}$ in rat platelet-rich plasma mirrors cerebral extracellular 5-HT levels, whilst 5-HT in isolated platelets mirrors neuronal 5-HT changes. Exp Brain Res 2002; 143: 191-7.

42 Ernst C, Wanner B, Brezo J, Vitaro F, Tremblay R, Turecki G. A deletion in tropomyosin-related kinase $\mathrm{b}$ and the development of human anxiety. Biol Psychiatry 2011; 69: 604-7.

43 Gross JA, Fiori LM, Labonté B, Lopez JP, Turecki G. Effects of promoter methylation on increased expression of polyamine biosynthetic genes in suicide. J Psychiatr Res 2013; 47: 513-9.

44 Labonte B, Yerko V, Gross J, Mechawar N, Meaney MJ, Szyf M, et al. Differential glucocorticoid receptor exon $1(B), 1(C)$, and $1(\mathrm{H})$ expression and methylation in suicide completers with a history of childhood abuse. Biol Psychiatry 2012; 72: 41-8.

45 Beach SRH, Brody GH, Lei MK, Gibbons FX, Gerrard M, Simons RL, et al. Impact of child sex abuse on adult psychopathology: a genetically and epigenetically informed investigation. J Fam Psychol 2013; 27: 3-11.

46 Vijayendran M, Beach SRH, Plume JM, Brody GH, Philibert RA. Effects of genotype and child abuse on DNA methylation and gene expression at the serotonin transporter. Front Psychiatry 2012; 3: 55

\section{Out-of-body experiences}

Peter Fenwick

Out-of-body experiences, in which the person feels they are viewing the world from outside their body, may be spontaneous or triggered by pain or fear, due to failure to integrate proprioceptive, tactile and visual information in the right parieto-temporal junction. They are similar to autoscopy, namely seeing your body in extra-personal space. But out-of-body experiences can occur in a near-death state during a cardiac arrest and be remembered even though brain processes are distorted or absent. Reliable accounts of patients who have acquired verifiable information while clinically dead suggest that consciousness may not after all be limited to the brain. 